\title{
PDK1 Gene
}

National Cancer Institute

\section{Source}

National Cancer Institute. PDK1 Gene. NCI Thesaurus. Code C104758.

This gene is involved in glucose metabolism. 